\title{
A TEST OF ALTERNATIVE HYPOTHESES FOR CHARACTER DIVERGENCE BETWEEN COEXISTING SPECIES
}

\author{
David W. Pfennig ${ }^{1}$ And Peter J. Murphy ${ }^{2}$ \\ Department of Biology, CB\#3280, University of North Carolina, Chapel Hill, North Carolina 27599-3280 USA
}

\begin{abstract}
Why do closely related, coexisting species typically differ in phenotypic features associated with resource use? One answer to this question is that such differences might generally evolve in allopatry, as different species adapt to divergent environmental conditions, and any differences that thereby accumulate might subsequently enable coexistence in sympatry. Alternatively, coexisting species might generally diverge in sympatry, because of selection to reduce competition for food (character displacement). Here we evaluated these two causes of character divergence by asking which hypothesis better explains differences in feeding morphology between tadpoles of two species of spadefoot toads, Spea bombifrons and S. multiplicata. We found that, in natural ponds containing both species, $S$. multiplicata almost always developed into a smaller, round-bodied tadpole with normal sized jaw muscles used for feeding on detritus at the pond bottom (the "omnivore" morph), whereas $S$. bombifrons almost always developed into a larger, flat-headed tadpole with greatly enlarged jaw muscles used for feeding on crustaceans in open water (the "carnivore" morph). By contrast, in all but one of 18 similar ponds containing a single species, both species expressed both phenotypes. Divergence between species in morph production appears to have evolved in sympatry: when we compared population means for each of four key trophic characters, we found that no allopatric population of S. bombifrons was as carnivore-like as the sympatric $S$. bombifrons, and, for three of four characters, no allopatric population of $S$. multiplicata was as omnivore-like as the sympatric $S$. multiplicata. In contrast to significant differences in trophic characters, we found no divergence between allopatric and sympatric populations in a character not directly involved in feeding on detritus or crustaceans (overall body size). These data, together with our earlier experimental work, reveal that coexisting $S$. bombifrons and $S$. multiplicata have diverged from one another in resource use and in phenotypic features associated with resource use because of selection to reduce competition for food (i.e., character displacement). Spea tadpoles, therefore, are one of few systems for which both experimental and observational evidence link phenotypic divergence to resource competition, thereby providing a model for understanding why coexisting species often differ phenotypically.
\end{abstract}

Key words: character displacement; competition; divergent selection; geographic variation; local adaptation; phenotypic plasticity; Spea bombifrons; Spea multiplicata.

\section{INTRODUCTION}

Natural communities typically comprise species that are more dissimilar in phenotypes than expected by chance (Brown 1995), and two distinct hypotheses have been advanced to explain this pattern (Rummel and Roughgarden 1985). First, species might adapt to different environmental conditions in allopatry, and these differences subsequently enable coexistence in sympatry (Grant 1972, Losos 1990, 1992, Thompson 1994). Second, competition might generate selection for the evolution of diverse phenotypes in sympatry ("character displacement": Darwin 1859, Lack 1947, Brown and Wilson 1956, Roughgarden et al. 1983, Schluter 1994, 2000, 2001). Establishing which of these hypotheses explains species divergence is central

Manuscript received 2 April 2002; revised 3 September 2002; accepted 25 September 2002. Corresponding Editor: B. Sinervo.

${ }^{1}$ E-mail: dpfennig@email.unc.edu

${ }^{2}$ Present address: Department of Biological Sciences, Idaho State University, Pocatello, Idaho 83209-8007 USA. for understanding the ecological origins of phenotypic diversity (Schluter 2000).

One way to test the above two hypotheses is to determine if coexisting species differ in phenotype only in sympatry or if they differ both in sympatry and in allopatry. The former pattern would implicate character displacement, whereas the latter would suggest that divergence may have arisen in allopatry before the species came together. Moreover, finding that species diverge only in traits associated with resource competition (e.g., trophic characters) would provide additional evidence for the character displacement hypothesis.

Here, we ask which of the above two competing hypotheses explains divergence between larvae of plains spadefoot toads (Spea bombifrons) and New Mexico spadefoot toads (S. multiplicata). Tadpoles of both species occur as two distinct morphs: (1) a smaller, slowly developing, round-bodied tadpole with normal sized jaw muscles and smooth mouthparts used for feeding on detritus at the pond bottom (the "omnivore" 
morph), and (2) a larger, more rapidly developing, narrow-bodied tadpole with greatly enlarged jaw muscles and notched mouthparts used for feeding on crustaceans in open water (the "carnivore" morph; Gilmore 1924, Smith 1934, Orton 1954, Bragg and Bragg 1958, Bragg 1965, Pomeroy 1981, Pfennig 1992a, $b$; see photos in Pfennig and Murphy 2002). Tadpoles are born as omnivores, but they may develop into carnivores following ingestion of anostracan fairy shrimp (Pomeroy 1981, Pfennig 1990).

In ponds where the two species co-occur, S. bombifrons are more prone to develop into carnivores (see Pfennig 1999), and resource competition appears to promote this divergence between species in carnivore morph production (Pfennig and Murphy 2000, 2002). In both field and laboratory populations, S. bombifrons (the superior competitor for shrimp) become increasingly more carnivore-like, whereas $S$. multiplicata (the superior competitor for detritus) become increasingly more omnivore-like, as the relative abundance of the other species is increased. Differences between species in morph production arise, in part, through phenotypic plasticity. In laboratory experiments, the presence of S. multiplicata facultatively enhances carnivore production among individual $S$. bombifrons tadpoles, whereas the presence of $S$. bombifrons facultatively suppresses carnivore production among individual $S$. multiplicata tadpoles (Pfennig and Murphy 2000). However, divergence also reflects genetically based canalized differences. At high elevations, where $S$. bombifrons are absent, $S$. multiplicata maintain plasticity to express the carnivore morphology, but at lower elevations, where S. bombifrons are present, S. multiplicata have evolved reduced propensities to express the carnivore morphology (Pfennig and Murphy 2000, 2002). Thus, selection imposed by competitors has led to the evolution of a canalized omnivore phenotype in S. multiplicata, which is distinct from the competitor's carnivore phenotype (Pfennig and Murphy 2000, 2002).

Although our earlier studies provide experimental evidence linking resource competition to morphological divergence, it remains unclear whether such divergence resulted from selection in sympatry to reduce resource competition (i.e., character displacement), or, alternatively, whether it was a carryover of differences that arose in allopatry as each species adapted to different environmental conditions in isolation from the other species. In order to distinguish between the above two possibilities, we compared trophic morphology in sympatry and in allopatry and used these data to infer whether any divergence in trophic morphology arose in sympatry only (as predicted by the character displacement model), both in sympatry and allopatry, or in allopatry only (Fig. 1a-d). Finding that all or part of the divergence arose in allopatry would suggest that each species adapted to different environmental conditions in allopatry, and that these differences subse-

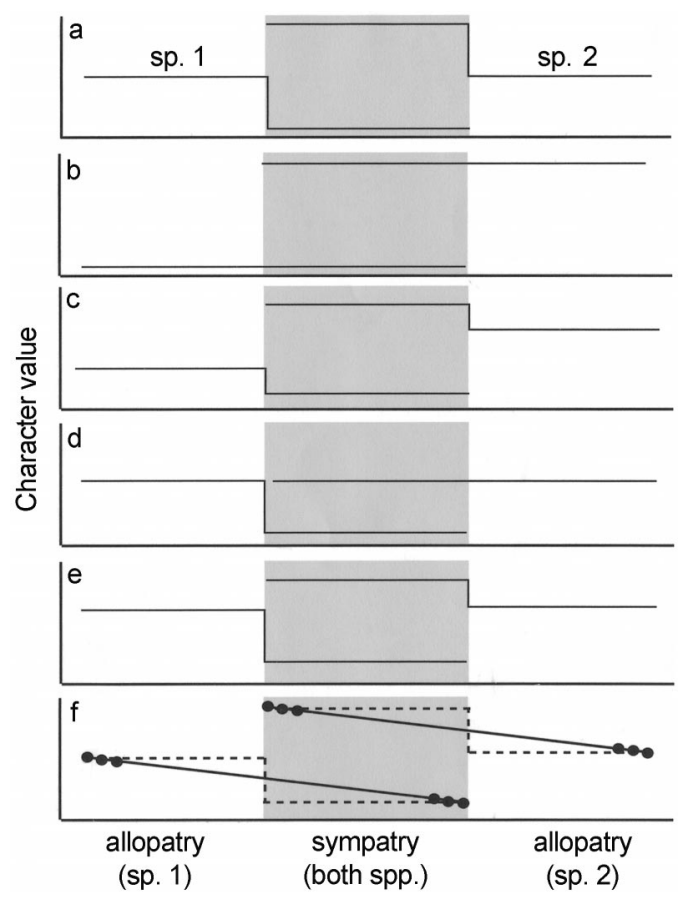

FIG. 1. Different hypotheses for character divergence between interacting species. Divergence can arise in (a) sympatry only (note that allopatric populations have identical character values), (b) allopatry only, with subsequent colonization of sympatric location, (c) both sympatry and allopatry, and (d) sympatry for one species only. Regardless of where divergence arises, it can be (a, c) symmetrical or (e) asymmetrical. In (f), note also that character displacement might be falsely inferred (dashed lines) if two species clines are roughly parallel but different in position, and if there is incomplete sampling (dots).

quently enabled species coexistence in sympatry (e.g., see Losos 1990, 1992). By contrast, finding that $S$. bombifrons are never as carnivore-like in allopatry as they are in sympatry, and finding that $S$. multiplicata are never as omnivore-like in allopatry as they are in sympatry, would suggest that species differences arose in sympatry (e.g., see Adams and Rohlf 2000, Losos 2000).

In addition, if we found evidence that divergence arose in sympatry, we sought to test the character displacement hypothesis in more detail by examining the following questions. First, did divergence occur in trophic characters only, and not in characters that were not directly involved in feeding on shrimp or detritus (e.g., overall body size)? Second, did the divergence in trophic characters follow a clinal gradient, suggesting that the differences observed in sympatry might have arisen in response to factors other than the presence of heterospecific competitors ("false" character displacement; Fig. 1f)? Finally, most cases of character displacement are asymmetrical, where one species changes more than the other (Schluter 2002). We evaluated whether Spea follow this pattern by asking whether divergence in Spea tadpoles is asymmetrical 


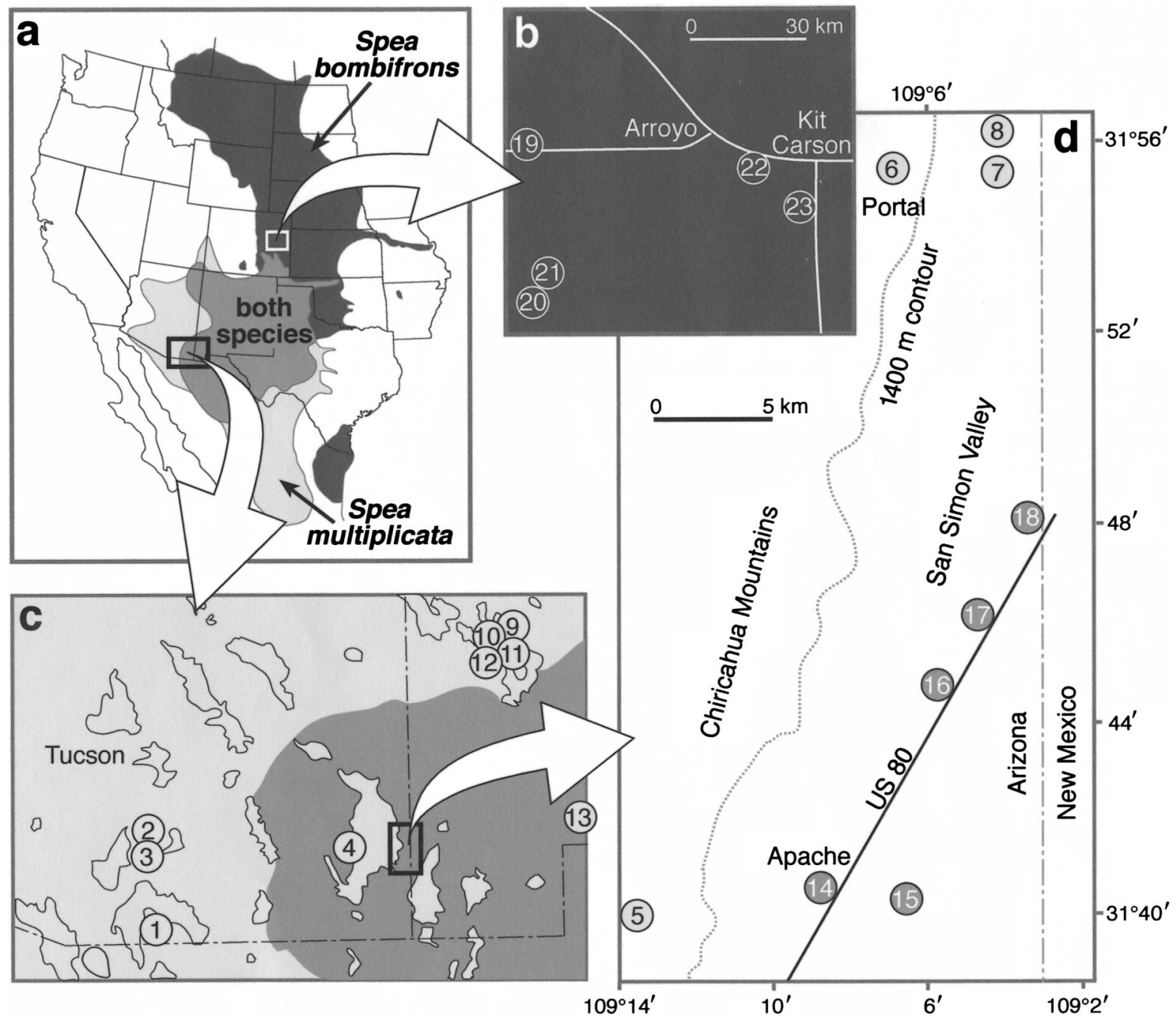

FIG. 2. (a) Geographical distributions of Spea bombifrons and S. multiplicata (from Conant and Collins 1998). (b) Locations of collection sites (encircled numbers) in eastern Colorado. (c) Geographical distributions of S. bombifrons and S. multiplicata in the basin and range province of southwestern New Mexico and southeastern Arizona showing locations of mountain ranges above $1400 \mathrm{~m}$ elevation and collection sites (encircled numbers). In the southeastern part of this region, only S. multiplicata is present above $1400 \mathrm{~m}$ (light gray), but both species are present at lower elevations (dark gray). To the west and north, only S. multiplicata is present at all elevations (light gray). (d) Map of the San Simon Valley of Arizona and New Mexico, showing locations of collection sites. In (b-d), light gray circles = ponds in which only S. multiplicata was present, medium gray circles $=$ ponds in which both species of Spea were present, and dark gray circles = ponds in which only S. bombifrons was present.

(Fig. 1e) or symmetrical (Fig. 1a, c) and addressing the possible selective basis of either pattern.

\section{Materials And Methods \\ General procedures}

In July 1999 and August 2001, we sampled Spea bombifrons and S. multiplicata tadpoles from 23 ponds using the methods in Pfennig (1990). Locations of study ponds are given in Fig. 2. We sampled both species in allopatry and sympatry and, within the zone of sympatry, we sampled ponds that historically have contained either one or both species. Regarding the latter types of ponds, in the basin and range province of southwestern New Mexico and southeastern Arizona, only S. multiplicata are present above $1400 \mathrm{~m}$, but both species are present at lower elevations (Simovich 1985; Fig. 2c). To the west and north, only $S$. multiplicata are present at all elevations (Fig. 2c, d).

Upon collection, tadpoles were scored as being omnivores or carnivores by visually assessing the shape of the head and mouthparts (morph assignments were unambiguous; e.g., see Pfennig 1992b, Pfennig and Murphy 2000). Tadpoles were then killed by immersion in a $0.1 \%$ aqueous solution of tricane methanesulfonate (MS 222, Sigma-Aldrich, St. Louis, Missouri), placed individually in a labeled plastic bag, and frozen at 
$-80^{\circ} \mathrm{C}$. Once we returned the tadpoles to the laboratory, we employed protein electrophoresis on tail tissue to classify tadpoles as $S$. bombifrons, S. multiplicata, or F1 hybrids (Simovich and Sassaman 1986). The remaining tadpole body was then transferred to $95 \%$ ethanol for morphometric analysis.

For morphometric analyses, we measured four trophic characters following the methods in Pfennig and Murphy (2002): (a) width of the orbitohyoideus $(\mathrm{OH})$ muscle, (b) width of the interhyoideus (IH) muscle, (c) shape of the keratinized mouthparts (MP), and (d) gut length (GL). Overall, based on our earlier studies (Pfennig 1992a, Pfennig and Murphy 2002), we expected more carnivorous tadpoles to show relatively larger $\mathrm{OH}$ and IH muscles, have MP with prominent serration, and possess shorter GLs for a given body size. Conversely, we expected tadpoles specializing on detritus to display relatively longer GL, relatively smaller $\mathrm{OH}$ and IH muscles, and MP without serration.

We measured the four trophic characters on 637 tadpoles from the 23 ponds (9-73 tadpoles per pond; for one population [pond 19], we had no measurements of IH and GL). We also measured the developmental stage (GS: Gosner 1960) and size (snout-vent length, SVL) of each tadpole prior to dissection. Before exploring statistical trends in tadpole morphology, we standardized the data (i.e., each character value for each tadpole) for body size (SVL), developmental stage (GS), and natal pond shrimp density (categorized at the time of tadpole sampling as high, medium, or low; for methods, see Pfennig 1990). We standardized the data for SVL and GS, because carnivorous morphs develop more rapidly than omnivorous morphs (Pfennig 1990, $1992 b$ ). We also standardized the data for shrimp density, because the density of shrimp in each pond affects the proportion of tadpoles in that pond that develop into carnivores (Pfennig 1990). To perform these standardizations, we combined all populations of both species and then regressed all values of each character (OH, IH, GL, and MP) separately on log SVL, GS, and shrimp density. Regressions produced normal residuals ( $\mathrm{OH}$ and $\mathrm{IH}$ were log transformed), which ensured that linear standardization did not introduce bias. We then derived a standardized value for each character and tadpole by adding each tadpole's residual back to the mean value for that character. We used pond means for analyses because, within a pond, tadpoles may compete for shrimp and detritus (Pfennig 1992b) and thus are not necessarily morphologically independent. These standardized morphological data were used in comparisons to answer the following four questions, which address both the cause of divergence in Spea tadpoles and describe the shape of the evolutionary response in both species.

\section{Question 1: Were there exaggerated differences in sympatry only?}

To address this question we looked at trophic morphology in S. bombifrons and S. multiplicata in sym- patry and allopatry both discretely, in terms of morph production (i.e., omnivores and carnivores), and continuously, in terms of the mean trophic character values (i.e., standardized values). For morph production, we compared the proportion of carnivores and omnivores produced by both species in sympatry and allopatry to determine whether the species diverged in morph production only in sympatry.

Next, to corroborate our comparisons based on discretely determined morphs, we used finer scale data on the trophic characters. First, we asked whether either species (or any region) tended to produce more exaggerated carnivores or omnivores. We were especially interested in knowing whether the most extreme carnivores were produced by $S$. bombifrons in sympatry, as previously suggested (e.g., see Orton 1954, Bragg and Bragg 1958, Bragg 1965). This was accomplished by comparing, qualitatively, the combined $z$ scores for $\mathrm{OH}$ and MP for each population to see if the values in any population exceeded those of all other populations (combining the $z$ scores for $\mathrm{OH}$ and MP served as a quantitative measure of the degree of carnivory; IH and GL were excluded from this analysis because of missing data for population 19).

Finally, for each species separately, we examined whether any allopatric population, on the basis of morphological similarity, could have served as the likely source of the sympatric populations. This was determined by comparing, qualitatively, the mean trait value for each allopatric population to see whether it equaled or exceeded the grand mean in sympatry. The existence of such divergent allopatric populations provides a mechanism other than character displacement for the origin of divergence in sympatry. Thus, we required two criteria be met for each trait to support a strong conclusion of sympatric divergence: (1) finding significant differences between sympatric, but not allopatric, populations of the two species, and (2) finding no allopatric populations of either species that equaled or exceeded the grand mean of their respective sympatric populations.

\section{Question 2: Were there exaggerated differences in trophic characters only?}

Next, we asked whether divergence occurred in trophic characters but failed to occur in characters that were not directly involved in feeding on shrimp or detritus, such as overall body size. To address this issue, we compared mean body size (SVL) in allopatric populations with that in sympatric populations by using a two-tailed $t$ test. We standardized SVL for GS by regressing SVL on GS.

\section{Question 3: Was there evidence of clinal divergence?}

We specifically asked if either species showed a clinal gradient toward becoming more omnivore-like (or more carnivore-like) as we gradually moved from the 


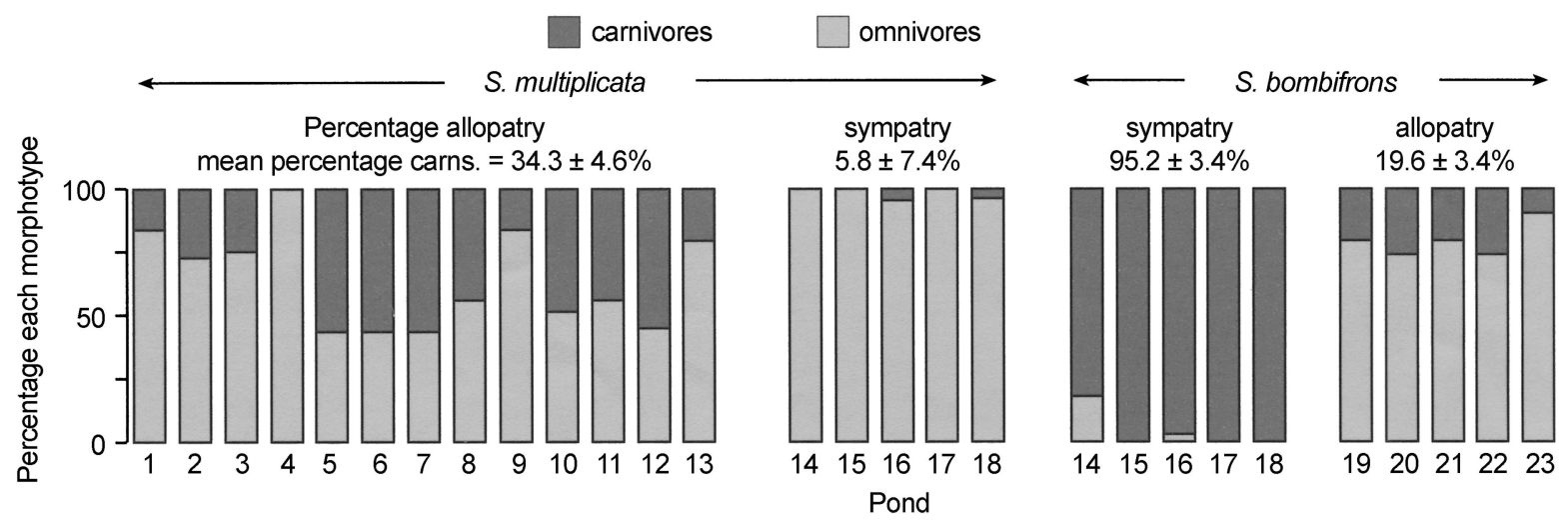

FIG. 3. Percentage of Spea multiplicata and S. bombifrons tadpoles expressing each morphotype in allopatric and sympatric ponds. The grand mean ( \pm 1 SEM) percentage of each species expressing the carnivore morphology in allopatry and sympatry is shown above each set of bars. Pond numbers correspond to those in Fig. 2.

zone of allopatry to the zone of sympatry. Such a pattern might suggest that the divergence arose in response to factors other than heterospecific competitors and would be indicative of false character displacement (sensu Endler 1986).

To address this question, we focused on one trait only, the width of the orbitohyoideus $(\mathrm{OH})$ muscle: $\mathrm{OH}$ is the character that best distinguishes carnivores from omnivores (Pfennig 1992b). We performed separate Spearman rank correlations for each species between the mean standardized $\mathrm{OH}$ values for each allopatric population and the longitude of the collection site (the data did not meet the requirements for parametric correlation). Data were available for five allopatric populations for S. bombifrons and 13 for S. multiplicata. We used these correlations to determine if (1) S. bombifrons became increasing more carnivore-like in more westerly populations (closer to sympatry; Fig. 2), and (2) S. multiplicata become increasing more omnivorelike in more easterly populations (closer to sympatry; Fig. 2).

\section{Question 4: Was divergence symmetrical or asymmetrical?}

To determine if one species was displaced consistently more than the other, we calculated for each species and each trait $(\mathrm{OH}, \mathrm{IH}, \mathrm{GL}$, and $\mathrm{MP})$ the mean percentage character change when going from allopatry to sympatry. Percentage character change was calculated as $(|\overline{\bar{A}}-\overline{\bar{S}}| \div \overline{\bar{A}}) \times 100$, where $\overline{\bar{S}}$ and $\overline{\bar{S}}$ represent the grand means of the allopatric and sympatric populations for each trait and species, respectively. We compared the mean arcsine square-root transformed percentage character change for each character across species by using a paired $t$ test and asked if one species was consistently displaced more than the other species.

RESUlts

\section{Question 1: Were there exaggerated differences in sympatry only?}

In general, the data met the first criterion for sympatric divergence: trophic characters of the two Spea species were significantly different from each other only in sympatry. Moreover, both species showed displacement from allopatry to sympatry.

First, when we compared the frequencies of carnivores and omnivores in different populations of both species, we found that in all five sympatric ponds sampled, the two species tended to be monomorphic. Spea multiplicata, in particular, almost always developed into omnivores, whereas $S$. bombifrons almost always developed into carnivores. By contrast, in 17 out of the 18 similar ponds containing a single species, both species were polymorphic and expressed both morphs (Fig. 3). Thus, the mean ( $\pm 1 \mathrm{SE}$ ) percentage of Spea multiplicata tadpoles expressing the carnivore morphology in allopatry $(34.3 \pm 4.6 \%)$ was significantly greater than that in sympatry $\left(5.8 \pm 7.4 \% ; t_{1,16}=3.3\right.$, $P<0.005$; Fig. 3), whereas the mean percentage of $S$. bombifrons carnivores in allopatry $(19.6 \pm 3.4 \%)$ was significantly less than in sympatry $\left(95.2 \pm 3.4 \% ; t_{1,8}\right.$ $=15.9, P<0.0001$; Fig. 3$)$. Moreover, the two species were markedly different in carnivore production in sympatry $\left(t_{1,8}=16.7, P<0.0001\right)$, but similar in allopatry $\left(t_{1,16}=1.7, P>0.1\right)$.

Interestingly, sympatric $S$. bombifrons were no more exaggerated in morphology than were many allopatric S. multiplicata carnivores (Fig. 4; compare the degree of carnivory of sympatric $S$. bombifrons with that of allopatric $S$. multiplicata in ponds 5 and 10). In addition, with the exception of one population (pond 14), $S$. multiplicata omnivores in sympatry were not more omnivore-like than S. multiplicata in allopatry (Fig. 4).

Next, we examined the tadpoles in more detail by comparing four key trophic characters. When sampled in allopatry, S. bombifrons and S. multiplicata were indistinguishable for three of the four trophic characters (Fig. 5). In particular, the two species did not differ in jaw musculature (mean \pm 1 SE orbitohyoideus width $[\mathrm{OH}]$ for $S$. bombifrons in allopatry $=1.477 \pm 0.056$ $\mathrm{mm}$ vs. for $S$. multiplicata in allopatry $=1.400 \pm 0.035$ $\mathrm{mm}, t_{1,16}=1.217, P=0.241$; Fig. 5a; mean inter- 


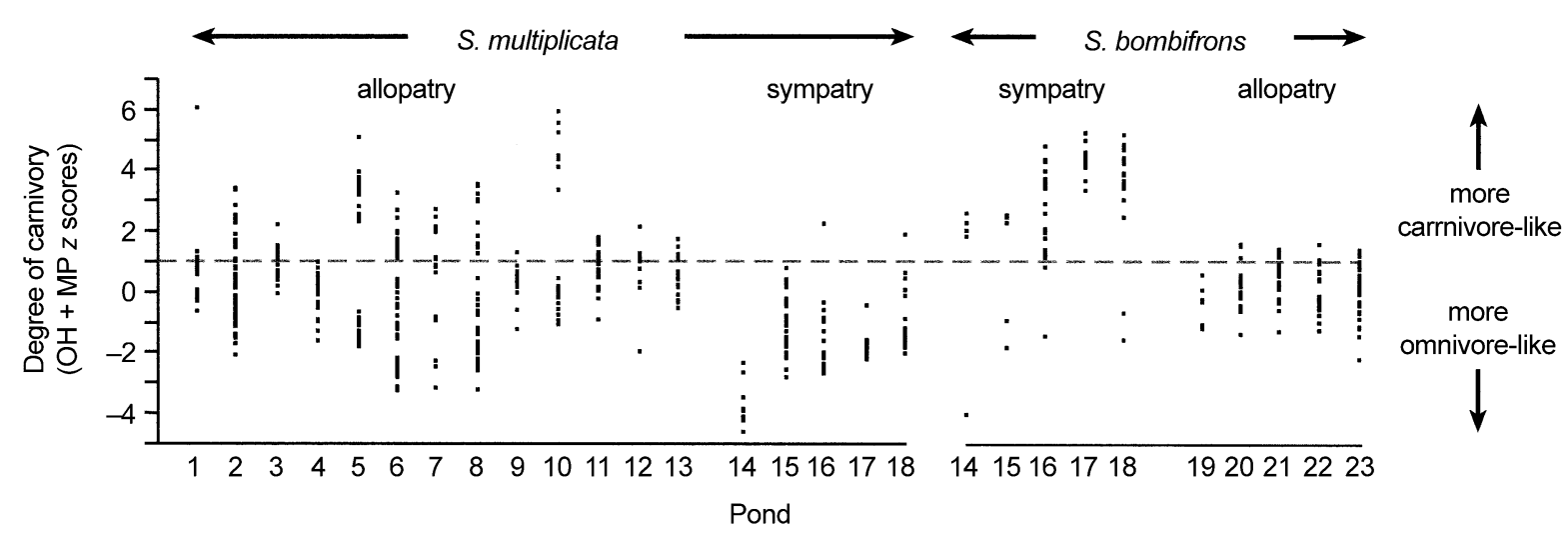

FIG. 4. Degree of carnivory, as measured by the combined $z$ scores for OH and MP, for Spea multiplicata and S. bombifrons tadpoles in allopatric and sympatric ponds. Each dot is the score for an individual tadpole. Pond numbers correspond to those in Fig. 2.

hyoideus width [IH] for S. bombifrons in allopatry = $0.581 \pm 0.028 \mathrm{~mm}$ vs. for $S$. multiplicata in allopatry $=0.592 \pm 0.015 \mathrm{~mm}, t_{1,15}=0.340, P=0.739$; Fig. $5 b$ ), nor did they differ in length of intestines (mean GL for $S$. bombifrons in allopatry $=147 \pm 15 \mathrm{~mm}$ vs. for $S$. multiplicata in allopatry $=158 \pm 9 \mathrm{~mm}, t_{1,15}=$ $0.620, P=0.545$; Fig. 5d). The two species did differ in allopatry in the shape of their mouthparts, with $S$. multiplicata expressing more carnivore-like features (mean MP score for $S$. bombifrons in allopatry $=1.943$ \pm 0.108 vs. for $S$. multiplicata in allopatry $=2.460 \pm$ 0.067, $t_{1,16}=4.081, P<0.001$; Fig. 5c).

In contrast to their overall similarity in allopatry, the two species were dissimilar, on average, for all four trophic structures in sympatry. In all cases, S. bombifrons tadpoles were more carnivore-like in the five ponds where the species co-occurred (Fig. 5). For instance, jaw muscles $(\mathrm{OH}$ and $\mathrm{IH})$ were significantly larger on $S$. bombifrons from sympatry $(\mathrm{OH}=1.586$ $\pm 0.111 \mathrm{~mm} ; \mathrm{IH}=0.727 \pm 0.057 \mathrm{~mm}$ ) than on $S$. multiplicata from the same populations $(\mathrm{OH}=1.025$ $\pm 0.111 \mathrm{~mm}, t_{1,8}=3.551, P<0.008 ; \mathrm{IH}=0.428 \pm$ $0.057 \mathrm{~mm}, t_{1,8}=3.687, P<0.007$; Fig. 3a, b), indicating that few $S$. multiplicata tadpoles possessed the large muscles necessary for capturing shrimp prey when $S$. bombifrons were present. Similarly, mouthparts (MP) were significantly more carnivore-like on $S$. bombifrons from sympatry $(3.667 \pm 0.275 \mathrm{~mm})$ than on $S$. multiplicata from sympatry $(1.430 \pm 0.275 \mathrm{~mm}$; $t_{1,8}=5.763, P<0.001$; Fig. 5c), indicating that few $S$. multiplicata tadpoles possessed the elaborate serrations necessary for capturing shrimp when $S$. bom-
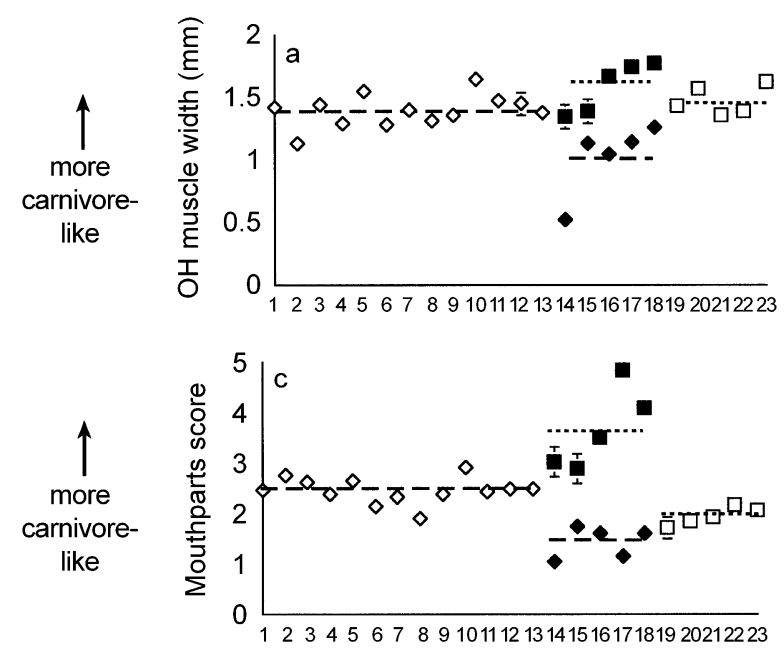
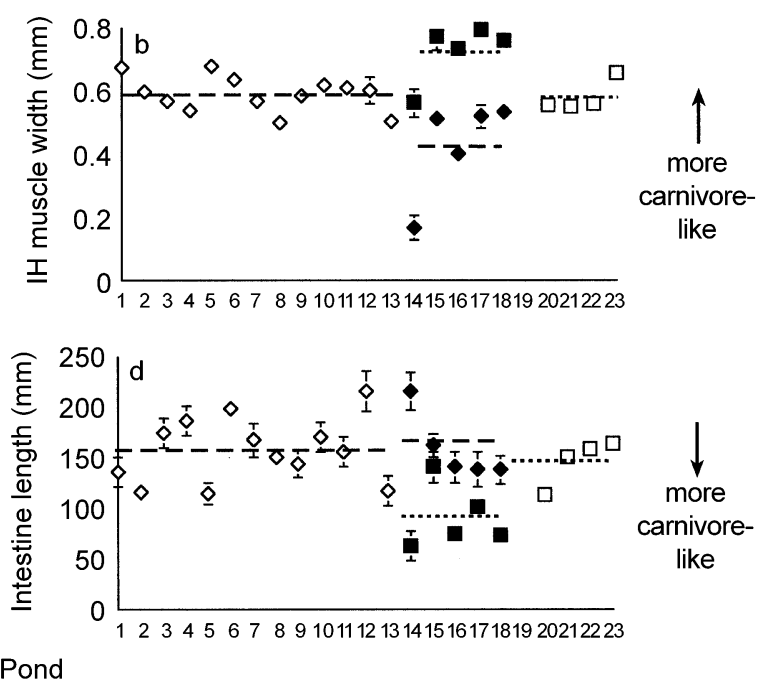

FIG. 5. Comparison of four trophic characters for Spea multiplicata (diamonds) and S. bombifrons (squares) in 13 ponds where only S. multiplicata occurred (ponds 1-13; open diamonds), five ponds where both species occurred together (ponds 14-18; closed symbols), and five ponds where only $S$. bombifrons occurred (ponds 19-23; open squares). Grand means for $S$. multiplicata and S. bombifrons are denoted by horizontal dashed and dotted lines, respectively. Pond numbers correspond to those in Fig. 2. 


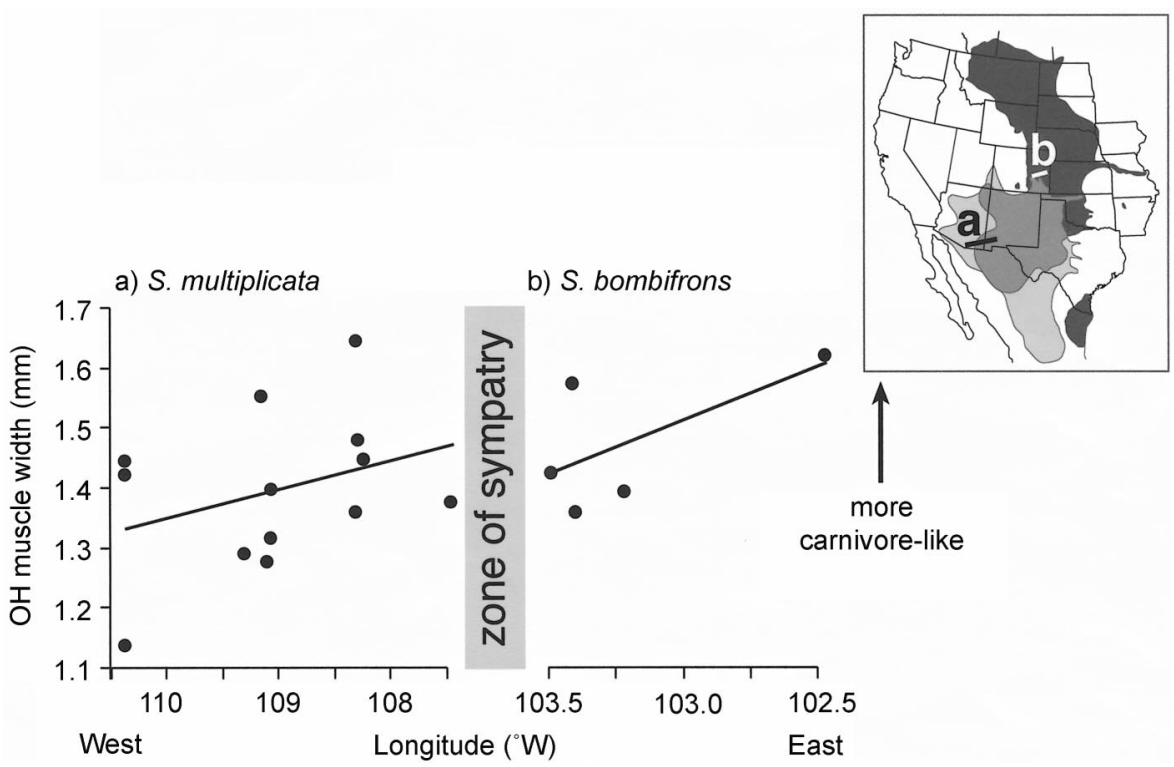

FIG. 6. Width of orbitohyoideus muscle as a function of longitude of natal pond for allopatric populations of (a) Spea multiplicata and (b) S. bombifrons. The least-squares regression line is shown for illustrative purposes only. Inset: geographical ranges of both species showing locations of sampling transects (solid line).

bifrons were present. Finally, intestines (GL) were significantly longer (i.e., more omnivore-like) in $S$. multiplicata from sympatry $(159 \pm 14 \mathrm{~mm})$ than in $S$. bombifrons from sympatry $\left(91 \pm 14 \mathrm{~mm} ; t_{1,8}=3.349\right.$, $P<0.011$; Fig. 5d), indicating that more $S$. multiplicata were equipped for processing detritus in sympatry. Moreover, not only were the two species significantly different in sympatry in terms of the means for each character, but, for three of the four characters $(\mathrm{OH}, \mathrm{IH}$, MP), the species means for the five different populations were completely non-overlapping (Fig. 5).

In comparisons within each species from allopatric to sympatric regions, we found that most trophic characters of both species exhibited displacement. Spea multiplicata tadpoles were significantly more omnivore-like in sympatry than in allopatry for three of four traits. In particular, $S$. multiplicata were significantly more omnivore-like for $\mathrm{OH}\left(t_{1,16}=3.877, P<0.002\right)$, IH $\left(t_{1,16}=3.410, P<0.004\right)$, and MP $\left(t_{1,16}=7.231\right.$, $P<0.001)$. Only GL did not differ significantly between regions $\left(t_{1,16}=0.088, P=0.931\right)$. In contrast, $S$. bombifrons were significantly more carnivore-like in sympatry than in allopatry. In particular, S. bombifrons were significantly more carnivore-like for IH $\left(t_{1,7}=2.762, P<0.028\right), \operatorname{MP}\left(t_{1,7}=4.634, P<0.002\right)$, and GL $\left(t_{1,7}=2.971, P<0.021\right)$. Only $\mathrm{OH}$ did not differ significantly between regions $\left(t_{1,7}=1.031, P=\right.$ $0.333)$.

In general, the morphological data also supported our second criterion for sympatric divergence, i.e., that no allopatric population of either species was displaced for a given character to a degree matching or exceeding the grand mean for that trait and respective species in sympatry. In looking across all four trophic characters for $S$. bombifrons, we found that none of the five allopatric populations were as carnivore-like as the grand mean of the five sympatric populations (Fig. 5). For $S$. multiplicata, we found that for three of the four characters $(\mathrm{OH}, \mathrm{IH}, \mathrm{MP})$ none of the 13 allopatric populations were as omnivore-like as the grand mean of the five sympatric populations (Fig. 5). Only GL in $S$. multiplicata deviated from this trend, where five of the 13 allopatric populations (ponds 3, 4, 6, 10, and 12 in Fig. $5 \mathrm{~d})$ were more omnivore-like than the sympatric grand mean.

\section{Question 2: Were there exaggerated differences in trophic characters only?}

In contrast to the significant differences in trophic characters, we found no differences between allopatric populations and sympatric populations of either species in overall body size (SVL). For $S$. multiplicata, the mean SVL in 13 allopatric populations was $12.652 \pm$ $1.216 \mathrm{~mm}$, whereas the mean SVL in five sympatric populations was $13.119 \pm 1.961 \mathrm{~mm}\left(t_{1,16}=0.202, P\right.$ $=0.842$ ). For $S$. bombifrons, the mean SVL in five allopatric populations was $14.216 \pm 1.187 \mathrm{~mm}$, whereas the mean SVL in five sympatric populations was $16.895 \pm 1.187 \mathrm{~mm}\left(t_{1,8}=1.596, P=0.149\right)$.

\section{Question 3: Was there evidence of clinal divergence?}

Neither species showed a significant correlation between the degree of carnivory (standardized $\mathrm{OH}$ ) and longitude (Fig. 6). No significant east to west trend was evident for either $S$. bombifrons $\left(r_{\mathrm{S}}=-0.314, n=5\right.$ 
FIG. 7. Percentage change for each of the four trophic characters by species. See Methods: Question 4, for calculation details. Percentage character change $=(|\overline{\bar{A}}-\overline{\bar{S}}| \div \overline{\bar{A}}) \times 100$ where $\overline{\bar{A}}$ and $\overline{\bar{S}}$ are the grand means of the allopatric and sympatric populations for each trait and species, respectively.

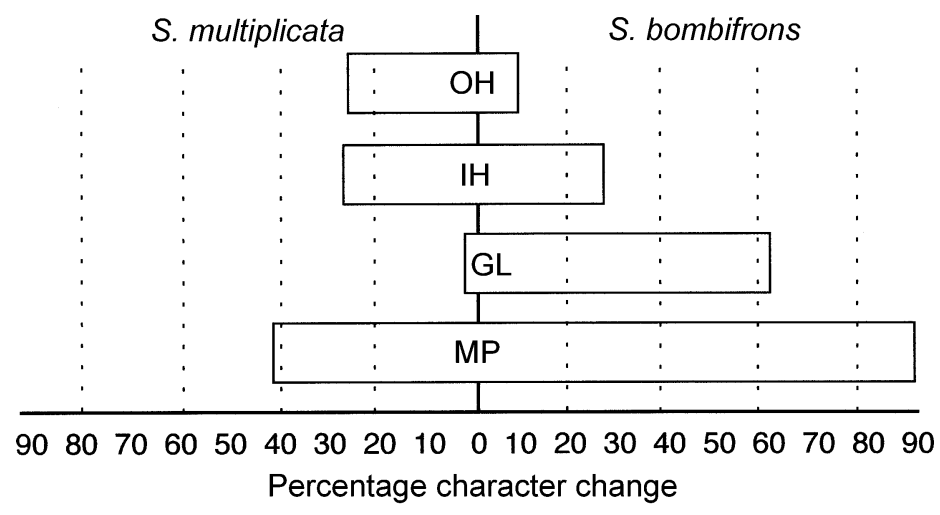

ponds, $P=0.544)$ or $S$. multiplicata $\left(r_{\mathrm{S}}=-0.290, n\right.$ $=13$ ponds, $P=0.337$ ). Indeed, the nonsignificant associations between $\mathrm{OH}$ and longitude were in the opposite direction to that predicted by the false character displacement hypothesis for both species. When we pooled both species and included the five sympatric populations, there was a nearly statistically significant trend for increasingly carnivorous features in eastern populations $\left(r_{\mathrm{S}}=-0.350, n=23\right.$ ponds, $\left.P=0.067\right)$.

\section{Question 4: Was divergence symmetrical or asymmetrical?}

Overall, neither species was displaced more than the other $\left(t_{1,3}=1.111, P=0.348\right)$. Spea multiplicata was displaced more in $\mathrm{OH}$, but $S$. bombifrons was displaced more in GL and MP. For IH, the displacement was approximately the same for $S$. bombifrons as for $S$. multiplicata (Fig. 7).

\section{DISCUSSION}

We sought to explore the selective bases of why closely related, coexisting species often differ phenotypically. Using two species of spadefoot toad tadpoles as a model system, we found that in ponds containing both species, Spea multiplicata tended to develop into a smaller tadpole that feeds on detritus at the pond bottom (the "omnivore" morph), whereas S. bombifrons tended to develop into a more robust tadpole that feeds on crustaceans in open water (the "carnivore" morph). Conversely, in ponds containing a single species, both species tended to express both morphs (Fig. $3)$. Thus, divergence in feeding morphology of Spea tadpoles follows the classic pattern of character displacement, where closely related species are recognizably different where they occur together but similar where each occurs alone (Brown and Wilson 1956; reviewed in Schluter 2000)

We evaluated two opposing hypotheses to explain this divergence in the expression of trophic polyphenism between sympatric $S$. bombifrons and $S$. multiplicata populations. In particular, we asked whether divergence resulted from selection in sympatry to reduce resource competition (i.e., character displacement), or, alternatively, whether it was a carryover of differences that arose in allopatry as each species adapted to different environmental conditions. Our results indicated that divergence most likely arose in sympatry and only occurred in trophic traits that directly influenced resource competition. Moreover, sympatric divergence did not result from false character displacement; we found no evidence for clinal divergence in $S$. bombifrons and $S$. multiplicata across the allopatric to sympatric regions. Finally, on balance, sympatric displacement was symmetric in tadpoles of both species. Below we discuss these results in turn and their broader implications.

Our results strongly suggest that divergence in expression of trophic polyphenism arose in sympatry, probably due primarily to competition for food among phenotypically similar tadpoles. Except for the shape of their mouthparts (MP), tadpoles from allopatric populations of $S$. bombifrons and $S$. multiplicata were not significantly different from each other in trophic morphology. Conversely, in sympatry, the species were significantly different for all four trophic characters.

Additional evidence indicates that neither an especially carnivorous lineage of $S$. bombifrons nor an especially omnivorous lineage of $S$. multiplicata gave rise to the divergent populations observed in sympatry. For $S$. bombifrons, when we compared population means for four critical trophic characters, we found that no allopatric population was as carnivore-like as the grand mean of populations in sympatry (Fig. 5). For S. multiplicata, in three of the four characters $(\mathrm{OH}, \mathrm{IH}, \mathrm{MP})$, none of the 13 allopatric populations (sampled from geographically diverse sites east and west of the sympatric ponds) were as omnivore-like as the grand mean of populations in sympatry (Fig. 5). Only GL in $S$. multiplicata deviated from this overall trend. Moreover, at a finer scale within the San Simon Valley of southeastern Arizona, S. multiplicata from mixed-species ponds were markedly more omnivore-like than $S$. multiplicata from pure-species ponds nearby $(<10 \mathrm{~km}$ distant; see Fig. 2d). Populations of S. multiplicata in neighboring pure and mixed species ponds likely share a common ancestry, implying that phenotypic diver- 
gence between populations in pure and mixed species ponds arose in sympatry.

Our evidence for sympatric divergence from field populations in this study bolsters our prior experimental findings (Pfennig and Murphy 2000, 2002). In those studies, we demonstrated that $S$. multiplicata became more omnivore-like in the presence of $S$. bombifrons, whereas $S$. bombifrons became more carnivore-like in the presence of S. multiplicata (Pfennig and Murphy 2000, 2002). Hence, both experimental and observational evidence indicate that these two species differ in phenotype because of a history of evolutionary adjustment by one species to the other, i.e., character displacement.

The present study also adds to the evidence that character displacement in Spea evolved from selection to minimize competition for food. In particular, we found that both species exhibited exaggerated divergence in sympatry in trophic characters only. In a character not directly related to competition for shrimp or detritus (overall body size), we uncovered no difference between sympatric and allopatric populations.

In addition to demonstrating that divergence in trophic morphology arose through resource competition in sympatry, our data appear to rule out false character displacement (Endler 1986). A clinal gradient in the propensity to express the carnivore morphology in both species, that is coincident with the patterns of overlap between the species, might suggest that the observed pattern of character displacement was an artifact of incomplete sampling (Fig. 1f). However, we found no evidence for such a gradient in either species. In particular, S. multiplicata did not become more omnivorelike as we moved from west to east in 13 sampled populations (i.e., as we moved from allopatry to sympatry; Fig. 6a). Similarly, in our more limited sample of five populations, $S$. bombifrons did not become more carnivore-like as we moved from east to west (i.e., as we moved from allopatry to sympatry; Fig. 6b). In fact, when the species data were pooled, the near-significant clinal trend was in the opposite direction to that predicted by false character displacement: $S$. multiplicata became more carnivore-like closer to sympatry, and $S$. bombifrons became more omnivore-like closer to sympatry (Fig. 6).

It is unclear why Spea should become more carnivore-like in more easterly populations (Fig. 6). One possible explanation for this pattern is that the costs associated with the omnivore phenotype may increase from west to east because of competition imposed by other species of anurans. As evidence for such a response, anuran species richness generally increases from west to east in the U.S. (e.g., see Fig. 12-3 in Duellman and Trueb 1986; for data on Colorado, see Fig. 2.6 in Hammerson 1999). More critically, all other species of anuran tadpoles in the central and eastern U.S. occupy the niche of the omnivore morph. Thus, in contrast to western populations, in eastern popula- tions, Spea tadpoles may be confronted with a greater array of omnivore-like competitors. These competitors might have imposed selection on Spea tadpoles in eastern populations, favoring the evolution of a more carnivore-like form.

Finally, we evaluated whether either species was displaced more than the other; i.e., whether divergence was symmetrical (Fig. 1a, c) or asymmetrical (Fig. 1e). Overall, neither species appeared to have been displaced more than the other. Spea multiplicata was displaced more in $\mathrm{OH}$, but $S$. bombifrons was displaced more in GL and MP (Fig. 7). Thus, we conclude that divergence was symmetrical overall, a pattern that differs from most other studies of character displacement (Schluter 2002). Yet, in contrast to symmetrical character displacement, asymmetrical displacement may generally be evolutionarily unstable, because, if greater divergence carries a greater fitness cost, then asymmetrical displacement might often ultimately lead to competitive exclusion of the species that undergoes greater divergence.

Our earlier studies revealed that character displacement in Spea tadpoles arises through both facultative changes in morphology and canalized differences in propensity to adopt the carnivore phenotype (Pfennig and Murphy 2000, 2002). Although competitively-mediated plasticity ("facultative character displacement"; Pfennig and Murphy 2002) has not traditionally been considered "true" character displacement (Grant 1972, Endler 1986, Schluter and McPhail 1992, Schluter 2000), because one of the six widely accepted criteria for character displacement is that phenotypic differences between populations and species should have a genetic basis (Grant 1972) — the magnitude and direction of a plastic response is often genetically variable and responsive to natural selection and evolutionary change (West-Eberhard 1989). Moreover, many plant and animal species use plasticity to respond to competitors adaptively (reviewed in Pfennig and Murphy 2002). Indeed, phenotypic plasticity is simply an alternative proximate mechanism-distinct from canalization-by which organisms can respond to competitors adaptively. Thus, cases of facultative character displacement that satisfy the other five criteria for character displacement (such as in S. multiplicata; Pfennig and Murphy 2000) should be regarded as "true" character displacement.

Additionally, facultative character displacement might represent an early stage in the evolution of "canalized character displacement", in which individuals in sympatry with competitors evolve a canalized phenotype that is unlike its competitor's phenotype (as observed in S. multiplicata; see Pfennig and Murphy 2000, 2002). Such canalization may occur, because as facultative character displacement causes one phenotype to be expressed continuously in a population (e.g., a phenotype unlike the competitor's phenotype), and as the alternative phenotype is never expressed, alleles 
that regulate expression of this "hidden" phenotype would not be exposed to selection, and thus are at risk of chance loss (e.g., through genetic drift or gradual mutation accumulation; West-Eberhard 1989). In this way, flexible alternative phenotypes may play a pivotal role as a phase of evolution that fosters diversification and accelerates change (West-Eberhard 2003).

In sum, our study links morphological divergence observed in nature directly to the mechanism of divergence, resource competition, demonstrated in past laboratory (Pfennig and Murphy 2000, 2002) and field (Pfennig 1992b) experiments. Our data therefore suggest that character displacement-phenotypic evolution stemming from resource competition between species-plays a central role in explaining why coexisting species often differ phenotypically.

\section{ACKNOWLEDGMENTS}

We thank K. Pfennig, W. Harcombe, and Vicki Derevyanny for assisting us with the research, the Southwestern Research Station of the American Museum of Natural History for furnishing housing and laboratory facilities, and K. Pfennig, K. Reeve, B. Sinervo, A. Welch, and two anonymous referees for commenting on the manuscript. We also gratefully acknowledge the Game and Fish Departments of Arizona, Colorado, and New Mexico for providing scientific collecting permits and the U.S. National Science Foundation for funding our research.

\section{Literature Cited}

Adams, D. C., and F. J. Rohlf. 2000. Ecological character displacement in Plethodon: biomechanical differences found from a geometric morphometric study. Proceedings of the National Academy of Sciences USA 97:4106-4111.

Bragg, A. N. 1965. Gnomes of the night: the spadefoot toads. University of Pennsylvania Press, Philadelphia, Pennsylvania, USA.

Bragg, A. N., and W. N. Bragg. 1958. Variations in the mouth parts in tadpoles of Scaphiopus (Spea) bombifrons Cope (Amphibia: Salientia). Southwestern Naturalist 3:55-69.

Brown, J. H. 1995. Macroecology. University of Chicago Press, Chicago, Illinois, USA.

Brown, W. L., and E. O. Wilson. 1956. Character displacement. Systematic Zoology 5:49-64.

Conant, R., and J. T. Collins. 1998. A field guide to reptiles and amphibians of eastern and central North America. Third edition. Houghton Mifflin, Boston, Massachusetts, USA.

Darwin, C. R. 1859. On the origin of species by means of natural selection. John Murray, London, UK.

Duellman, W. E., and L. Trueb. 1986. Biology of amphibians. McGraw-Hill, New York, New York, USA.

Endler, J. A. 1986. Natural selection in the wild. Princeton University Press, Princeton, New Jersey, USA.

Gilmore, R. J. 1924. Notes on the life history and feeding habitats of the spadefoot toad of the western plains. Colorado College Publications Science Series 13:1-12.

Gosner, K. L. 1960. A simplified table for staging anuran embryos and larvae with notes on identification. Herpetologica 16:183-190.

Grant, P. R. 1972. Convergent and divergent character displacement. Biological Journal of the Linnean Society 4: $39-68$.

Hammerson, G. A. 1999. Amphibians and reptiles in Colorado. Second edition. University Press of Colorado, Niwot, Colorado, USA.

Lack, D. 1947. Darwin's finches. Cambridge University Press, Cambridge, UK.
Losos, J. B. 1990. A phylogenetic analysis of character displacement in Caribbean Anolis lizards. Evolution 44:558569.

Losos, J. B. 1992. The evolution of convergent structure in Caribbean Anolis communities. Systematic Biology 41:403420.

Losos, J. B. 2000. Ecological character displacement and the study of adaptation. Proceedings of the National Academy of Sciences USA 97:5693-5695.

Orton, G. L. 1954. Dimorphism in larval mouthparts in spadefoot toads of the Scaphiopus hammondi group. Copeia 1954:97-100.

Pfennig, D. W. 1990. The adaptive significance of an environmentally-cued developmental switch in an anuran tadpole. Oecologia 85:101-107.

Pfennig, D. W. 1992a. Proximate and functional causes of polyphenism in an anuran tadpole. Functional Ecology 6: 167-174.

Pfennig, D. W. 1992b. Polyphenism in spadefoot toad tadpoles as a locally-adjusted evolutionarily stable strategy. Evolution 46:1408-1420.

Pfennig, D. W. 1999. Cannibalistic tadpoles that pose the greatest threat to kin are most likely to discriminate kin. Proceedings of the Royal Society of London Series B 266: 57-61.

Pfennig, D. W., and P. J. Murphy. 2000. Character displacement in polyphenic tadpoles. Evolution 54:1738-1749.

Pfennig, D. W., and P. J. Murphy. 2002. How fluctuating competition and phenotypic plasticity mediate species divergence. Evolution 56:1217-1228.

Pomeroy, L. V. 1981. Developmental polymorphism in the tadpoles of the spadefoot toad Scaphiopus multiplicatus. Dissertation. University of California, Riverside, California, USA.

Roughgarden, J., D. Heckel, and E. R. Fuentes. 1983. Coevolutionary theory and the biogeography and community structure of Anolis. Pages 371-410 in R. B. Huey, E. R. Pianka, and T. W. Schoener, editors. Lizard ecology: studies of a model organism. Harvard University Press, Cambridge, Massachusetts, USA.

Schluter, D. 1994. Experimental evidence that competition promotes divergence in adaptive radiation. Science 266: 798-801.

Schluter, D. 2000. The ecology of adaptive radiation. Oxford University Press, Oxford, UK.

Schluter, D. 2001. Ecological character displacement. Pages 265-276 in C. W. Fox, D. A. Roff, and D. J. Fairbairn, editors. Evolutionary ecology: concepts and cases studies. Oxford University Press, Oxford, UK.

Schluter, D. 2002. Character displacement. Pages 149-150 in M. Pagel, editor. Encyclopedia of evolution. Oxford University Press, Oxford, UK.

Schluter, D., and J. D. McPhail. 1992. Ecological character displacement and speciation in sticklebacks. American Naturalist 140:85-108.

Simovich, M. A. 1985. Analysis of a hybrid zone between the spadefoot toads Scaphiopus multiplicatus and Scaphiopus bombifrons. Dissertation. University of California, Riverside, California, USA.

Simovich, M. A., and C. A. Sassaman. 1986. Four independent electrophoretic markers in spadefoot toads. Journal of Heredity 77:410-414.

Smith, H. M. 1934. The amphibians of Kansas. American Midland Naturalist 15:377-528.

Thompson, J. N. 1994. The coevolutionary process. Chicago University Press, Chicago, Illinois, USA.

West-Eberhard, M. J. 1989. Phenotypic plasticity and the origins of diversity. Annual Review of Ecology and Systematics 20:249-278.

West-Eberhard, M. J. 2003. Developmental plasticity and evolution. Oxford University Press, Oxford, UK. 